\title{
Simultaneous interrelationship between the oral health behavior and oral health status of mothers and their children
}

\author{
Mitsugi Okada ${ }^{1)}$, Makoto Kawamura ${ }^{2)}$, Yoko Hayashi ${ }^{3)}$, Naoko Takase ${ }^{4)}$ and \\ Katsuyuki Kozai ${ }^{3)}$ \\ ${ }^{1)}$ Department of Special Care Dentistry, Hiroshima University Hospital, Hiroshima, Japan \\ ${ }^{2)}$ Department of Preventive Dentistry, Hiroshima University Hospital, Hiroshima, Japan \\ ${ }^{3)}$ Department of Pediatric Dentistry, Graduate School of Biomedical Sciences, Hiroshima University, \\ Hiroshima, Japan \\ ${ }^{4)}$ Research and Development Department, Sunstar Inc., Takatsuki, Japan
}

(Received 15 May and accepted 22 October 2008)

\begin{abstract}
The purpose of this study was to examine the simultaneous interrelationships of oral health behavior and oral health between mothers and their children using the linear structural relations (LISREL) program. The subjects comprised 117 pairs of mothers and their children aged between 8 and 11 years. Dental status was expressed as DMFT. The Oral Rating Index (ORI) for mothers and ORI for children (ORI-C) were used as measures of gingival health. Hiroshima University Dental Behavioral Inventory (HU-DBI) was used for the assessment of mothers' oral health behavior. HU-DBI had a direct positive relationship to ORI, a direct negative relationship to DFT, and a direct positive association with ORI-C. Toothbrushing had a direct negative relationship to ORI-C. The hypothesized model was found to be closely consistent with the data. These results indicate that the periodontal health status of mothers is directly related to the oral health status of their children, and that toothbrushing by children is directly related to their gingival health. (J. Oral Sci. $50,447-452,2008$ )
\end{abstract}

Keywords: oral health attitudes/behavior; mothers/ children; linear structural relations (LISREL); factor analysis.

Correspondence to Dr. Mitsugi Okada, Department of Special Care Dentistry, Hiroshima University Hospital, 1-2-3 Kasumi, Minami-ku, Hiroshima 734-8553, Japan

Tel: +81-82-257-5788

Fax: +81-82-257-5789

E-mail: mitsugi@hiroshima-u.ac.jp

\section{Introduction}

Many studies have addressed the relationships between children's oral health behavior and oral health, and their mothers' or parents' socioeconomic status, health beliefs and oral health behavior. Maternal support is essential for prevention of dental caries and gingivitis in children among families with parents. It has been shown in a previous study that for children at the ages of 5 to 6 , a more positive maternal attitude is related to a lower incidence of caries in the child, better child oral hygiene, and more dental treatment received by the child (1). Furthermore, the gingival condition of mothers as a result of their oral health behavior has been shown to be associated with the prevalence and severity of dental caries in their 3-year-old children (2). However, little is known about mother-related factors associated with the oral health of schoolchildren at different stages of their development $(3,4)$.

It has been reported that, although schoolchildren believe that appropriate behavior can promote health, they do not develop an effective awareness of this relationship until the third to fourth grade (5). From the viewpoint of Bandura's social cognitive theory (6), the overt behavior of significant others represents an important source of social influence. Socialization involving oral health behavior may be considered a modeling process in which children imitate the behavior of their parents, who are available and valued role models for their offspring. Hence, the present study was performed to test the hypothesis that mothers' oral health behavior and periodontal health influence the oral health (dental caries and gingivitis) of their children 
throughout the period of growth and development.

The linear structural relations (LISREL) program provides an opportunity to evaluate an entire set of relationships at the same time (7). It has several advantages over more traditional statistical methods, particularly as it explores the causal links rather than mere empirical relationships between variables. In addition, knowledge of the methodological adequacy of the data-gathering process and the quality of measurement instruments can be directly incorporated into LISREL models by estimating the proportion of variance in an indicator, i.e. error variance.

The purpose of the present study was to examine the simultaneous interrelationships among mothers' oral health behavior and their periodontal health, and their children's oral health behavior and oral health status.

\section{Theoretical model}

The construction of the hypothetical model was based on the findings of previous studies of factors affecting oral health (1-4, 8-13). First, it was hypothesized that mothers' oral health behavior or oral health status is directly or indirectly linked to their children's oral health status (caries prevalence and gingival health) through children's oral health behavior. Second, it was hypothesized that dental caries in schoolchildren is causally linked to their gingival health level, which reflects oral hygiene status (self-care level).

\section{Subjects and Methods}

The subjects comprised 117 pairs of mothers and their children aged between 8 and 11 years, attending Hiroshima University Dental Hospital, Hiroshima City, Japan. Consent for participation was obtained prior to enrollment from the mothers of all children in the present study. The Hiroshima University Dental Behavioral Inventory (HU-DBI) (14) was used for assessment of the mothers' oral health behavior. The maximum score of the HU-DBI is 12 . This index has been shown to be internally consistent (Cronbach's alpha $=0.76)(15)$ and to have a good test-retest reliability (0.73) over a 4-week period (16).

Children were also asked about their oral health behavior through a questionnaire comprising 30 items. When a child agreed, partly agreed, partly disagreed, and disagreed, we assigned a score of 1, 2, 3, and 4, respectively. From the answers given, lower scores indicated better oral health behavior of children. Oral examinations were conducted at the Pediatric Dental Clinic. The Oral Rating Index for Children (ORI-C), which consists of five categories $(+2$ : excellent, +1 : good, 0 : questionable, -1 : poor, and -2 : very poor) was used for gingival health examination as described previously (17). It was performed by a trained pediatric dentist (MO), using an artificial light with a set of standard photographs of each level of the scale to maintain consistent standards. The children were then dentally examined by a second trained pediatric dentist ( $\mathrm{YH}$ ), using the WHO caries diagnostic criteria for DMFT (18). The dental examinations took place with the subjects supine using an artificial light, a dental explorer and a dental mirror. The ORI (19), which evaluates gingival heath care and oral hygiene level in adults, consists of five categories $(+2$ : excellent, +1 : good, 0 : questionable, -1 : poor, and -2 : very poor). This was followed by a promax rotation of the factors that accounted for the greatest amount of variation and computation of factor loadings for each question to identify any that exceeded 0.45 , which was used as a threshold for moderate to high loadings. An item was assigned to factor $\mathrm{X}$ when its absolute value of loading was more than 0.45 and it had no loading at 0.35 or higher for another factor.

Descriptive statistics and Pearson's correlation were used to provide preliminary information about the associations between six selected parameters. The overall fit was assessed by three measures: the goodness of fit index (GFI), the adjusted goodness of fit index (AGFI) and the root mean square error of approximation (RMSEA). In this study, the quality of model fit was considered reasonable, the probability of both GFI and AGFI being greater than 0.90, and RMSEA being less than 0.05.

Statistical analyses were conducted using SPSS 10.0J and Amos 4.0 (SPSS Inc., Chicago, IL, USA; SmallWaters Co., Chicago, IL, USA).

\section{Results}

From the promax answers, 6 items were extracted, dental anxiety (DA), desire to improve oral care (DIOC), toothbrushing (TB), dependency on snacks (DOS), persistence (PER), and concern over the number of cavities (CONC) in Table 1. The DA category was drawn from the answers to 2 questions, DIOC from the answers to a further 4, TB from 3 questions, DOS from 3 questions, PER from 2 questions, and CONC from the answers to 2 questions. For each item, the proportion of "appropriate" responses was calculated. The "appropriate" responses were determined through consideration of current information about the topic addressed by the item. No question was used to estimate response for more than one of the 6 selected items.

Table 2 shows the descriptive statistics and correlation among eleven selected variables.

The outline of the proposed model is given in Fig. 1. Analysis suggested that overall our model was satisfactory $(\mathrm{GFI}=0.949, \mathrm{AGFI}=0.917, \mathrm{RMSEA}=0.000) . \mathrm{HUDBI}$ 
Table 1 Extracted factors on promax answers

\begin{tabular}{|c|c|c|c|c|c|c|}
\hline Questionnaire & DA & DIOC & TBS & DOS & PER & CONC \\
\hline 21. In the waiting room, I worry about what my treatment would be like. & 0.91 & -0.02 & -0.09 & -0.12 & -0.07 & 0.05 \\
\hline 15. I am usually getting nervous on the day before visiting a dentist. & 0.84 & -0.26 & -0.02 & 0.08 & 0.02 & -0.08 \\
\hline 22. I want to know how to use dental floss properly. & -0.13 & 0.67 & 0.03 & -0.06 & 0.15 & -0.02 \\
\hline 28. I want to know the risk of getting my own cavities. & 0.04 & 0.67 & -0.04 & 0.03 & -0.25 & 0.02 \\
\hline 12. I want to know the proper way to brush my teeth. & -0.06 & 0.66 & 0.26 & -0.12 & 0.00 & -0.07 \\
\hline 4. After brushing my teeth I look at them in a mirror. & -0.06 & -0.13 & 0.83 & 0.10 & -0.10 & -0.03 \\
\hline 9. I make sure to brush my teeth clean one by one. & 0.06 & 0.06 & 0.75 & -0.20 & -0.01 & 0.06 \\
\hline 11. My dentist tells me I am brushing my teeth well. & -0.09 & 0.15 & 0.74 & 0.07 & -0.02 & 0.08 \\
\hline 26. I often have opportunities to eat snacks at home or outside. & -0.01 & 0.00 & 0.07 & 0.83 & -0.05 & -0.02 \\
\hline 20. In my free time I often tend to eat snacks unconsciously. & -0.05 & 0.00 & 0.07 & 0.82 & -0.05 & 0.01 \\
\hline 14. I often skip a meal because I ate too much snacks. & -0.04 & -0.12 & -0.16 & 0.63 & 0.17 & 0.27 \\
\hline 17. Usually when I start something I never finish it. & 0.20 & -0.09 & 0.07 & -0.02 & -0.79 & 0.05 \\
\hline 1. I remember that I have had many cavities since I recognized them. & -0.07 & -0.07 & 0.11 & -0.01 & -0.05 & 0.85 \\
\hline 6. I have new cavities arise no matter how many times I go to the dentist. & 0.04 & -0.01 & -0.04 & 0.13 & -0.07 & 0.71 \\
\hline
\end{tabular}

DA: dental anxiety; DIOC: desire of improving oral care; TBS: toothbrushing situation; DOS: dependency on snacks; PER: persistence; CONC: concern over number of cavities

Table 2 Descriptive statistics and correlation among eleven selected variables

\begin{tabular}{|c|c|c|c|c|c|c|c|c|c|c|c|}
\hline & DA & DIOC & TBS & DOS & PER & CONC & $\mathrm{dft}$ & DFT & ORI-C & ORI & HU-DBI \\
\hline $\mathrm{N}$ & 117 & 117 & 117 & 117 & 117 & 117 & 117 & 117 & 117 & 117 & 117 \\
\hline Mean & 0.00 & 0.00 & 0.00 & 0.00 & 0.00 & 0.00 & 2.79 & 0.34 & 0.50 & 0.50 & 5.89 \\
\hline S.D. & 1.00 & 1.00 & 1.00 & 1.00 & 1.00 & 1.00 & 3.26 & 0.81 & 0.91 & 1.01 & 2.22 \\
\hline DA & 1 & & & & & & & & & & \\
\hline DIOC & $0.22^{*}$ & 1 & & & & & & & & & \\
\hline TBS & 0.16 & $0.22^{*}$ & 1 & & & & & & & & \\
\hline DOS & 0.17 & 0.06 & -0.11 & 1 & & & & & & & \\
\hline PER & 0.08 & 0.11 & $0.39^{* * *}$ & -0.13 & 1 & & & & & & \\
\hline CONC & 0.17 & 0.06 & $-0.19^{*}$ & 0.04 & -0.16 & 1 & & & & & \\
\hline $\mathrm{dft}$ & -0.16 & -0.10 & 0.10 & $0.20^{*}$ & 0.10 & $-0.26^{* * *}$ & 1 & & & & \\
\hline DFT & 0.00 & 0.02 & 0.16 & -0.18 & 0.14 & $-0.34^{* * *}$ & 0.01 & 1 & & & \\
\hline ORI-C & 0.01 & -0.13 & $-0.26^{* *}$ & -0.04 & -0.10 & 0.05 & 0.04 & 0.02 & 1 & & \\
\hline ORI & -0.04 & -0.04 & -0.02 & 0.04 & 0.12 & 0.10 & 0.01 & $-0.20^{*}$ & $0.19^{*}$ & 1 & \\
\hline HU-DBI & -0.02 & 0.07 & -0.01 & 0.02 & 0.04 & 0.09 & -0.11 & 0.08 & 0.03 & $0.27^{* *}$ & 1 \\
\hline
\end{tabular}

Abbreviations: see Table 1. *: $P<0.05 ; * *: P<0.01 ; * * *: P<0.001$

had a direct positive relationship (path) to ORI $(0.27, P<$ 0.01 ). ORI had a direct negative relationship (path) to DFT $(-0.20, P<0.05)$ and a direct positive relationship (path) to ORI-C $(0.19, P<0.05)$. TB had a direct negative relationship (path) to ORI-C $(-0.26, P<0.01)$. DFT had a direct negative relationship (path) to CONC $(-0.33, P<$ 0.001 ). The dft had a direct positive relationship (path) to DOS $(0.20, P<0.05)$ and two direct negative relationships (path) to CONC $(-0.26, P<0.01)$ and DA $(-0.21, P<0.05)$, respectively. In addition, dft had an indirect positive relationship (path) to DIOC $(0.21, P<0.05)$ through DA. Moreover, DIOC had a direct positive relationship (path) to TB $(0.19, P<0.05)$. PER and DIOC had a direct positive relationship (path) to TB $(0.37, P<0.001$ and $0.19, P<$ 0.05 , respectively).

\section{Discussion}

This is the first known model to have revealed a simultaneous interrelationship between the oral health behavior and gingival health status of mothers, and the oral 
health behavior and gingival health of their children using LISREL analysis. The obtained data were factor-analyzed using promax rotation that provided an oblique solution, as opposed to varimax rotation that gives a rectangular solution. Varimax rotation is often used to obtain factors without mutual correlation, whereas oblique rotation offers solutions that recognize the relevance between mutual factors. As it is generally accepted that naturally occurring phenomena are never totally unrelated, oblique rotation has been suggested to produce results that are more practical. When considering relationships, it should be borne in mind that a significant correlation coefficient is not generally proof of causality, and that the relationships obtained are only correlative. Therefore, a behavioral model for explaining oral health behavior and gingival health between mothers and their children was tested using this program.

As results of this analysis, it appeared that mothers' gingival health level (ORI) had a direct influence to both caries experience (DFT) and the gingival health level (ORI-C) of their children. Furthermore, children's toothbrushing had a direct effect on their gingival health level. It was suggested that these findings support the necessity of continued emphasis on mothers' self-care strategies for not only their gingival health but also their children's oral health condition (dental caries and gingival health). The relationship between mothers' gingival health level and caries prevalence in their children in the present study was in agreement with the findings of some previous reports $(1,2)$. In addition, a significant correlation between parents' oral health behavior and their children's dental health behavior has been found (9). Moreover, children's persistence in their individual characters influences their gingival health through toothbrushing. Therefore, it is encouraging that clinicians, co-dental staff, schoolteachers, and parents can improve the gingivitis status of children through toothbrushing motivation, concerning their individual characters. Also, mothers' oral health status is essential for successfully solving the issue $(1,2)$. Behavioral management for mothers and children is required to establish their oral self-care along with oral hygiene instruction. Better attention should be paid to not only the child's oral health care but also that of their mothers. Therefore, mothers' own oral behavior is deemed to be

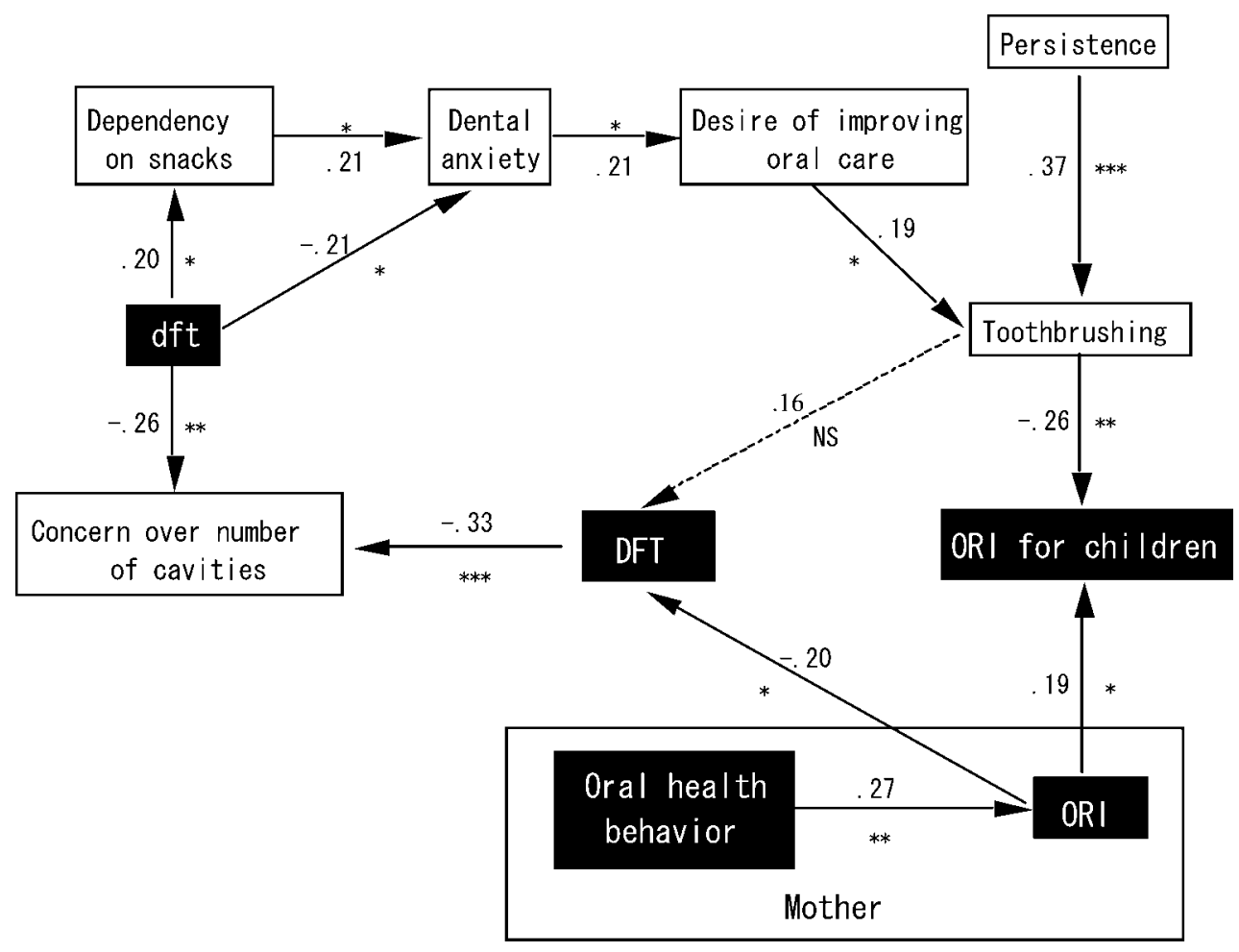

Fig. 1 Outline of the model.

ORI: Oral Rating Index; DFT: Decayed Teeth and Filled Teeth; dft: Decayed Teeth and Filled Teeth among primary teeth. The estimates are displayed in the path diagram: standardized regression weights near single-headed arrows. Statistical significance, *: $P<0.05$, **: $P<0.01$, ***: $P<0.001$, NS: Not significant. 
especially important.

It is of great interest that children with a high prevalence of caries in primary teeth also showed higher dental anxiety, lower dependency on snacks, and higher concern over the number of cavities. In addition, in the present study, children with a high caries experience in permanent teeth also demonstrated higher concern for the number of cavities. In subjects 15 to 18 years of age, dental anxiety is likely to be a significant predictor of dental caries, and may also be a risk factor for dental caries incidence (20). Many reports have indicated that caries experience in children is associated with toothbrushing, flossing, sweets, and regular dental check-ups $(9,10,21)$. Unexpectedly, the results of the present study indicated that children with high caries experience tended to report avoiding confectionery more often. This may be related to caries experience of the present subjects, aged 8 to 11 years, at younger ages or their mothers' educational attitude for preventing new caries. Further longitudinal studies are required to understand this observed trend in sweet preference.

In the present study, the apparent effect of mothers' oral health behavior on the gingival health status of their children was not statistically significant. For most Japanese mothers, the extent to which they check their children's teeth and oral hygiene gradually decreases until the child starts secondary or high school (22). It is suggested that children aged 10 and over could begin to take responsibility for their own gingival health as a natural step in their growth and development (4). In this study, there was no obvious correlation between dft and ORI-C, and DFT. Mean scores for dft and DFT in the subjects were 2.79 and 0.34 , respectively. For children attending regular checkups, this might be a result of the control (or reduction) in risk factors for dental caries in permanent teeth where there had been experience of caries in the primary dentition. A previous report showed that in children aged 7 to 12 years, oral hygiene was associated with both decayed teeth and gingival health status (13). The inconsistency may be due to the subjects' age, living in an urban or rural area, or the opportunity for prevention offered by regular dental checkups.

In conclusion, it was found that mothers' periodontal health level had a significant direct relationship to the oral health status of their school-aged children, and that children's toothbrushing had a significant direct relationship to their gingival health.

\section{References}

1. Sarnat H, Kagan A, Raviv A (1984) The relation between mothers' attitude toward dentistry and the oral status of their children. Pediatr Dent 6, 128-131
2. Sasahara H, Kawamura M, Kawabata K, Iwamoto Y (1998) Relationship between mothers' gingival condition and caries experience of their 3-year-old children. Int J Paediatr Dent 8, 261-267

3. Okada M, Kawamura M, Miura K (2001) Influence of oral health attitude of mothers on the gingival health of their school aged children. ASDC J Dent Child 68, 379-383

4. Okada M, Kawamura M, Kaihara Y, Matsuzaki Y, Kuwahara S, Ishidori H, Miura K (2002) Influence of parents' oral health behaviour on oral health status of their school children: an exploratory study employing a causal modelling technique. Int $\mathrm{J}$ Paediatr Dent 12, 101-108

5. Chang C, Chen LH, Chen PY (1994) Developmental stages of Chinese children' concepts of health and illness in Taiwan. Zhonghua Min Guo Xiao Er Ke Yi Xue Hui Za Zhi 35, 27-35

6. Bussey K, Bandura A (1984) Influence of gender constancy and social power on sex-linked modeling. J Pers Soc Psychol 47, 1292-1302

7. Jöreskog KG (1978) Structural analysis of covariance and correlation matrices. Psychometrika 43, 443-477

8. Sallis JF, Nader PH (1988) Family determinants of health behaviors. In Health behavior: emerging research perspectives, Gochman DS ed, Springer, New York, 107-126

9. Åstrøm AN, Jakobsen R (1996) The effect of parental dental health behavior on that of their adolescent offspring. Acta Odontol Scand 54, 235-241

10. Stewart JE, Jacobs Schoen M, Padilla MR, Maeder LA, Wolfe GR, Harts GW (1991) The effect of a cognitive behavioral intervention on oral hygiene. J Clin Periodontol 18, 219-222

11. McCaul KD, Glasgow RE, Gustafson C (1985) Predicting levels of preventive dental behaviors. $\mathrm{J}$ Am Dent Assoc 111, 601-605

12. Tedesco LA, Keffer MA, Fleck-Kandath C (1991) Self-efficacy, reasoned action, and oral health behavior reports: a social cognitive approach to compliance. J Behav Med 14, 341-355

13. Okada M, Kuwahara S, Kaihara Y, Ishidori H, Kawamura M, Miura K, Nagasaka N (2000) Relationship between gingival health and dental caries in children aged 7-12 years. J Oral Sci 42, 151155

14. Kawamura M, Iwamoto Y, Wright FAC (1997) A comparison of self-reported dental health attitudes and behavior between selected Japanese and Australian students. J Dent Educ 61, 354-360

15. Kawamura M (1988) Dental behavioral science -The 
relationship between perceptions of oral health and oral status in adults-. Hiroshimadaigaku Shigaku Zasshi 20, 273-286 (in Japanese)

16. Kawabata K, Kawamura M, Miyagi M, Aoyama H, Iwamoto Y (1990) The dental health behavior of university students and test-retest reliability of the HU-DBI. Koku Eisei gakkai Zasshi 40, 474-475 (in Japanese)

17. Okada M, Kuwahara S, Kozai K, Kawamura M, Nagasaka N (1999) The efficacy of an oral rating index for children for screening gingival health and oral hygiene status. Pediatr Dent J 9, 91-97

18. World Health Organization (1987) Individual tooth status and treatment need. In Oral health surveys: basic methods, 3rd ed, World Health Organization, Geneva, 34-39

19. Kawamura M, Fukuda S, Inoue C, Sasahara H,
Iwamoto Y (2000) The validity and reproducibility of an oral rating index as a measurement of gingival health care and oral hygiene level in adults. J Clin Periodontol 27, 411-416

20. Kruger E, Thomson WM, Poulton R, Davies S, Brown RH, Silva PA (1998) Dental caries and changes in dental anxiety in late adolescence. Community Dent Oral Epidemiol 26, 355-359

21. Miyazawa H, Lin Y-F, Eda S, Iwasaki H, Ueda T, Nakamura H, Tonomura M (1994) Primary environment factors and gingivitis in children -relationship between life custom and gingivitisShoni Shikagaku Zasshi 32, 801-810 (in Japanese)

22. Taura K (1981) Relationship between dental caries in deciduous teeth and tooth brushing in nursery school children. Koku Eisei gakkai Zasshi 31, 174188 (in Japanese) 